\title{
Differential expression of MAGI2-antisense RNA 3 in cancers of the breast.
}

Shahan Mamoor, MS ${ }^{1}$

$3 \quad$ shahanmamoor@gmail.com

East Islip, NY 11730

Breast cancer affects women at relatively high frequency ${ }^{1}$. We mined published microarray datasets ${ }^{2,3}$ to determine in an unbiased fashion and at the systems level genes most differentially expressed in the primary tumors of patients with breast cancer. We report here significant differential expression of the gene encoding MAGI2-antisense RNA 3, MAGI2-AS3, when comparing primary tumors of the breast to the tissue of origin, the normal breast. MAGI2-AS3 was also differentially expressed in the tumor cells of patients with triple negative breast cancer. MAGI2-AS3 mRNA was present at significantly lower quantities in tumors of the breast as compared to normal breast tissue. Analysis of human survival data revealed that expression of MAGI2-AS3 in primary tumors of the breast was correlated with distant metastasis-free survival in patients with basal-like subtype cancer, demonstrating a relationship between primary tumor expression of a differentially expressed gene and patient survival outcomes influenced by PAM50 molecular subtype. MAGI2-AS3 may be of relevance to initiation, maintenance or progression of cancers of the female breast.

Keywords: breast cancer, MAGI2-AS3, MAGI2-antisense RNA 3, systems biology of breast cancer, targeted therapeutics in breast cancer. 
Invasive breast cancer is diagnosed in over a quarter of a million women in the United States each year $^{1}$ and in 2018 , breast cancer was the leading cause of cancer death in women worldwide ${ }^{4}$. While patients with localized breast cancer are provided a 99\% 5-year survival rate, patients with regional breast cancer, cancer that has spread to lymph nodes or nearby structures, are provided an $86 \% 5$-year survival rate $^{5,6}$. Patients with metastasis to distant sites, like the brain, are provided a $27 \% 5$-year survival rate ${ }^{5,6}$. Understanding how primary tumors are most transcriptionally different from the tissue from which they originate, the breast, can facilitate development of novel diagnostic and therapeutics to promote early detection and enhanced treatment, and contribute to efforts to prevent progression to metastatic stages. We mined published microarray data ${ }^{2,3}$ to understand at the transcriptome level and in an unbiased fashion genes most differentially expressed in primary tumors of the breast as compared to normal breast tissue. MAGI2-antisense RNA 3 emerged as among the most differentially expressed genes in cancer of the female breast.

\section{Methods}

We utilized datasets GSE42568 ${ }^{2}$ and GSE $38959^{3}$ for this global differential gene expression analysis of female breast cancer. GSE42568 was generated using Affymetrix Human Genome U133 Plus 2.0 array technology with $n=17$ normal breast tissue biopsies and $n=104$ primary breast tumor biopsies from patients with breast cancer; analysis was performed using platform GPL570; the majority of patients whose tumors were analyzed were age 50 or older. GSE38959 was generated using Agilent-014850 Whole Human Genome Microarray 4x44K G4112F technology with $n=13$ samples of normal mammary gland ductal cells and $n=30$ samples of tumor cells from patients with triple negative breast cancer; analysis was performed using platform GPL4133. The tissues whose expression was profiled in this dataset are paired tissues ( 25 tumors matching 25 breast tissues from 25 patients). The Benjamini and Hochberg method of $p$-value adjustment was used for ranking of differential expression but raw $p$-values were used to assess statistical significance of global differential expression. Log-transformation of data was auto-detected, and the NCBI generated category of platform annotation was used. A statistical test was performed to evaluate whether MAGI2-AS3 expression was significantly different between primary breast tumors and normal breast tissue using a two-tailed t-test. For Kaplan-Meier survival analysis, we used the Kaplan-Meier plotter online tool ${ }^{7}$ for correlation of MAGI2-AS3 mRNA expression levels with distant metastasis-free survival (DMFS) in $n=283$ patients with basal-like subtype cancer, $n=259$ patients with luminal A subtype cancer, $n=183$ patients with luminal B subtype cancer, $n=218$ patients with HER2+ cancer, and $n=15$ patients with normal-like subtype cancer.

\section{Results}

We performed discovery of genes associated with breast cancer in females by mining two independently published microarray datasets ${ }^{2,3}$.

\section{MAGI2-AS3 is differentially expressed in primary tumors of the breast.}

Studying the global gene expression profiles of 104 breast cancers from patients aged 31 to 89 revealed that the gene encoding MAGI2-antisense RNA 3, MAGI2-AS3, was among the genes most differentially expressed in tumors of the breast in human breast cancer (Chart 1). When sorting each of the genes expressed in tumors of the breast based on significance of difference as compared to normal breast tissue, MAGI2-AS3 ranked 42 out of 54675 total transcripts, equating to $99.9 \%$ differential expression (Chart 1). Differential expression of MAGI2-AS3 in female breast cancer was statistically significant (Chart $1 ; p=5.48 \mathrm{E}-35)$. 
Analysis of a second microarray dataset ${ }^{3}$, here studying global gene expression patterns in the tumor cells of patients with triple negative breast cancer (TNBC), again revealed significant differential expression of MAGI2-AS3 in human breast cancer (Chart 2). When sorting each of the genes expressed in the tumors of patients with breast cancer (TNBC) based on significance of difference as compared to normal breast tissue, MAGI2-AS3 ranked 2473 out of 45015 total transcripts, equating to $94.5 \%$ differential expression (Chart 2). Differential expression of MAGI2-AS3 in the tumor cells of patients with TNBC was statistically significant (Chart $2 ; p=1.29 \mathrm{E}-05)$. These data suggested that differential expression of MAGI2-AS3 was not an artifact of a single microarray dataset, nor was it strictly associated with one type of breast cancer, rather a general feature of cancers of the breast.

\section{MAGI2-AS3 is expressed at significantly lower levels in breast tumors as compared to the breast.}

We obtained exact mRNA expression levels for MAGI2-AS3 from the breast and from breast tumors to understand the magnitude and direction of MAGI2-AS3 expression change. MAGI2-AS3 was expressed at lower levels in tumors of the breast as compared to normal breast tissue (Figure 1). Decreased expression of MAGI2-AS3 in primary breast tumors was statistically significant (Figure 1: $p<0.0001$ ). MAGI2-AS3 was expressed at $6.40 \pm 2.01$ arbitrary units (AU) in normal breast tissue, while it was expressed at $2.56 \pm 0.43 \mathrm{AU}$ in tumors of the breast. We calculated a mean fold change of $0.40 \mathrm{in}$ MAGI2-AS3 mRNA levels when comparing primary tumors of the breast to normal breast tissues.

\section{MAGI2-AS3 expression correlates with survival outcomes in basal-like subtype human breast cancer.}

We performed Kaplan-Meier survival analysis to study relationships between tumor MAGI2-AS3 mRNA expression levels and survival outcomes in patients with breast cancer. We observed a correlation between MAGI2-AS3 expression and distant metastasis-free survival (DMFS) in patients with basal-like subtype breast cancer which trended towards statistical significance (Figure 2; log rank $p$-value: 0.086 for distant metastasis-free survival, hazard ratio: 0.67 (0.42-1.06) (Fig. 2)). MAGI2-AS3 mRNA levels were a positive prognostic indicator in basal-like subtype breast cancer patients. Median DMFS, on average, markedly shorter for basal-like patients with low tumor expression of MAGI2-AS3 as compared to basal-like patients with high tumor expression of MAGI2-AS3 (Chart 3). MAGI2-AS3 primary tumor expression was not correlated with distant metastasis-free survival in luminal A breast cancer (Figure 2; log rank $p$-value: 0.17 for DMFS, hazard ratio: 1.62 (0.81-3.23) (Fig. 2)), luminal B subtype breast cancer (Figure 2; log rank $p$-value: 0.43 for DMFS, hazard ratio: 1.28 (0.69-2.34) (Fig. 2)), in HER2+ breast cancer (Figure 2; log rank $p$-value: 0.62 for DMFS, hazard ratio: 1.13 (0.7-1.82) (Fig. 2)) or in patients with normal-like breast cancer (Figure 2; log rank $p$-value: 0.88 for DMFS, hazard ratio: 1.13 (0.23-5.63) (Fig. 2)).

Thus, through comparative transcriptome analysis of primary tumors of the breast and normal breast tissue, we found that differential expression and down-regulation of MAGI2-AS3 was among the most significant transcriptional features in primary tumors from patients with breast cancer. MAGI2-AS3 expression in primary tumors of the breast was correlated with distant metastasis-free survival in patients with basal-like subtype disease, with mRNA levels of MAGI2-AS3 a positive prognostic indicator for basal-like breast cancer patients.

\section{Discussion}

Invasive breast cancer is a medical problem with a $27 \% 5$-year survival rate for women whose disease has spread to distant sites ${ }^{5,6}$. To facilitate understanding of the basic transcriptional differences between primary tumors of the breast and the tissues from which these tumors originate, normal breast 


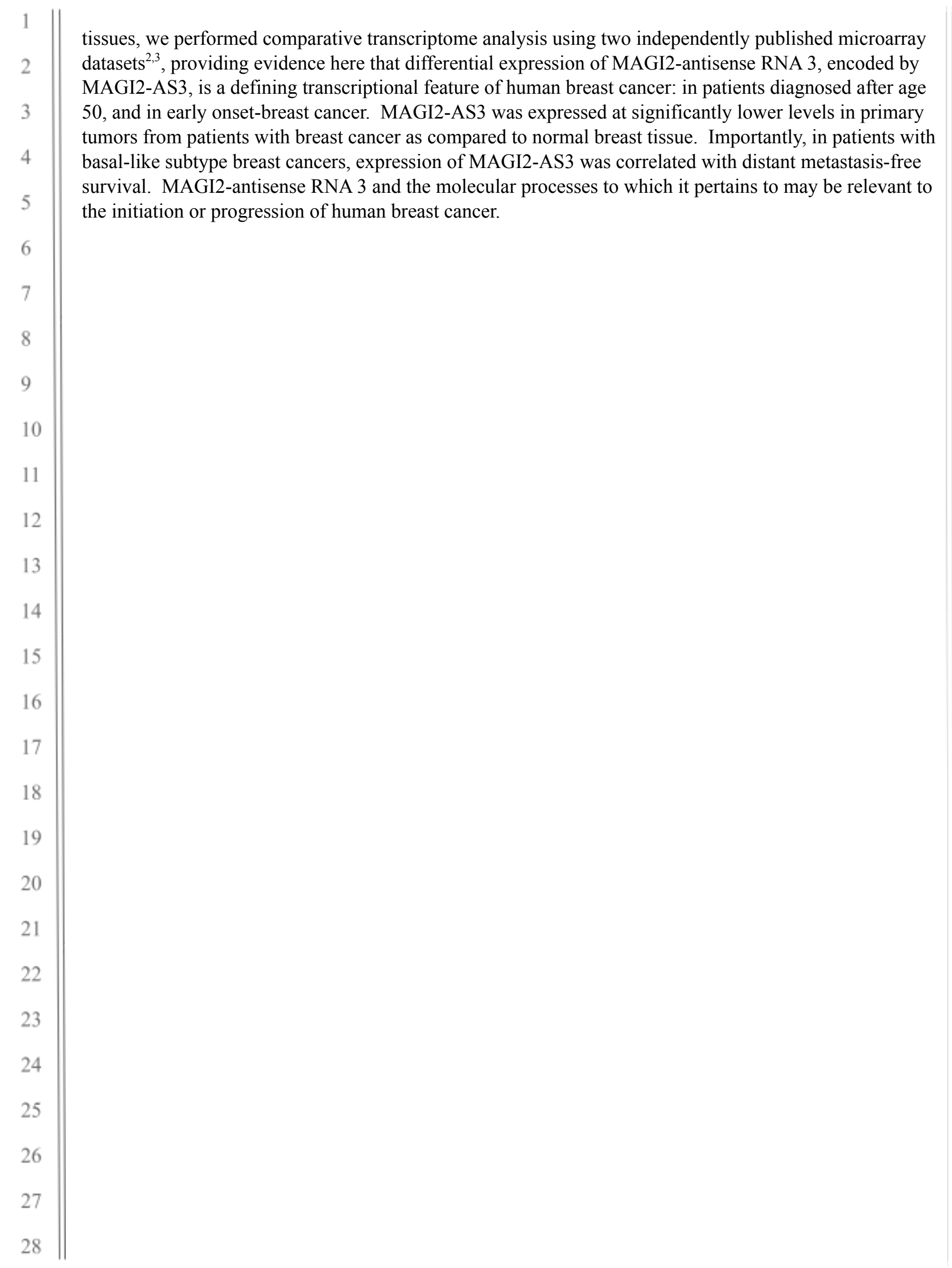




\section{References}

1. DeSantis, C.E., Ma, J., Goding Sauer, A., Newman, L.A. and Jemal, A., 2017. Breast cancer statistics, 2017, racial disparity in mortality by state. CA: a cancer journal for clinicians, 67(6), pp.439-448.

2. Clarke, C., Madden, S.F., Doolan, P., Aherne, S.T., Joyce, H., O’driscoll, L., Gallagher, W.M., Hennessy, B.T., Moriarty, M., Crown, J. and Kennedy, S., 2013. Correlating transcriptional networks to breast cancer survival: a large-scale coexpression analysis. Carcinogenesis, 34(10), pp.2300-2308.

3. Komatsu, M., Yoshimaru, T., Matsuo, T., Kiyotani, K., Miyoshi, Y., Tanahashi, T., Rokutan, K., Yamaguchi, R., Saito, A., Imoto, S. and Miyano, S., 2013. Molecular features of triple negative breast cancer cells by genome-wide gene expression profiling analysis. International journal of oncology, 42(2), pp.478-506.

4. Bray, F., Ferlay, J., Soerjomataram, I., Siegel, R.L., Torre, L.A. and Jemal, A., 2018. Global cancer statistics 2018: GLOBOCAN estimates of incidence and mortality worldwide for 36 cancers in 185 countries. CA: a cancer journal for clinicians, 68(6), pp.394-424.

5. ACS Cancer Facts \& Figures 2019.

https://www.cancer.net/cancer-types/breast-cancermetastatic/statistics.

6. Survival Rates for Breast Cancer. https://www.cancer.org/cancer/breast-cancer/ understanding-a-breast-cancer-diagnosis/breast-cancer-survival-rates.html

7. Györffy, B., Lanczky, A., Eklund, A.C., Denkert, C., Budczies, J., Li, Q. and Szallasi, Z., 2010. An online survival analysis tool to rapidly assess the effect of 22,277 genes on breast cancer prognosis using microarray data of 1,809 patients. Breast cancer research and treatment, 123(3), pp.725-731. 


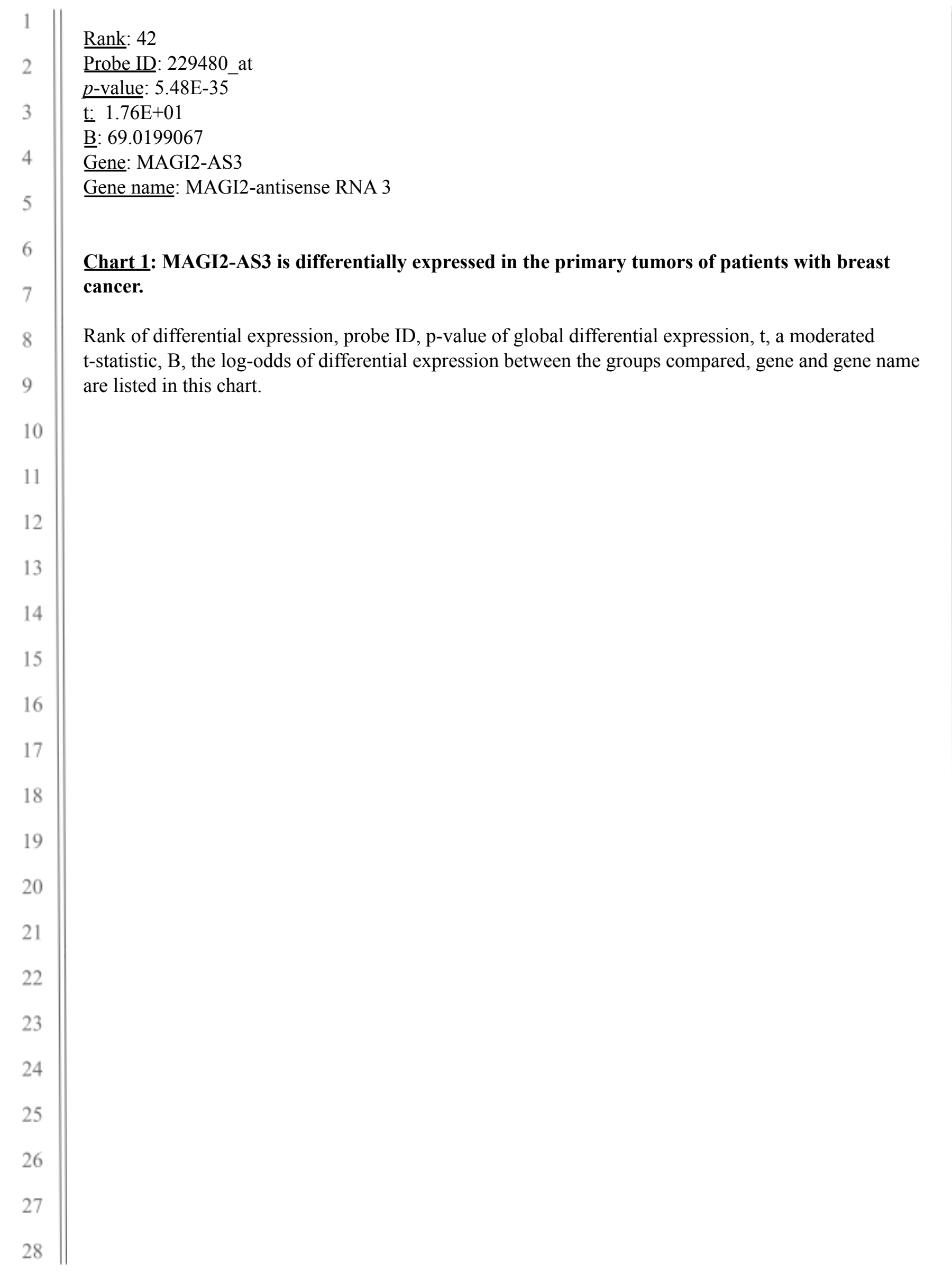




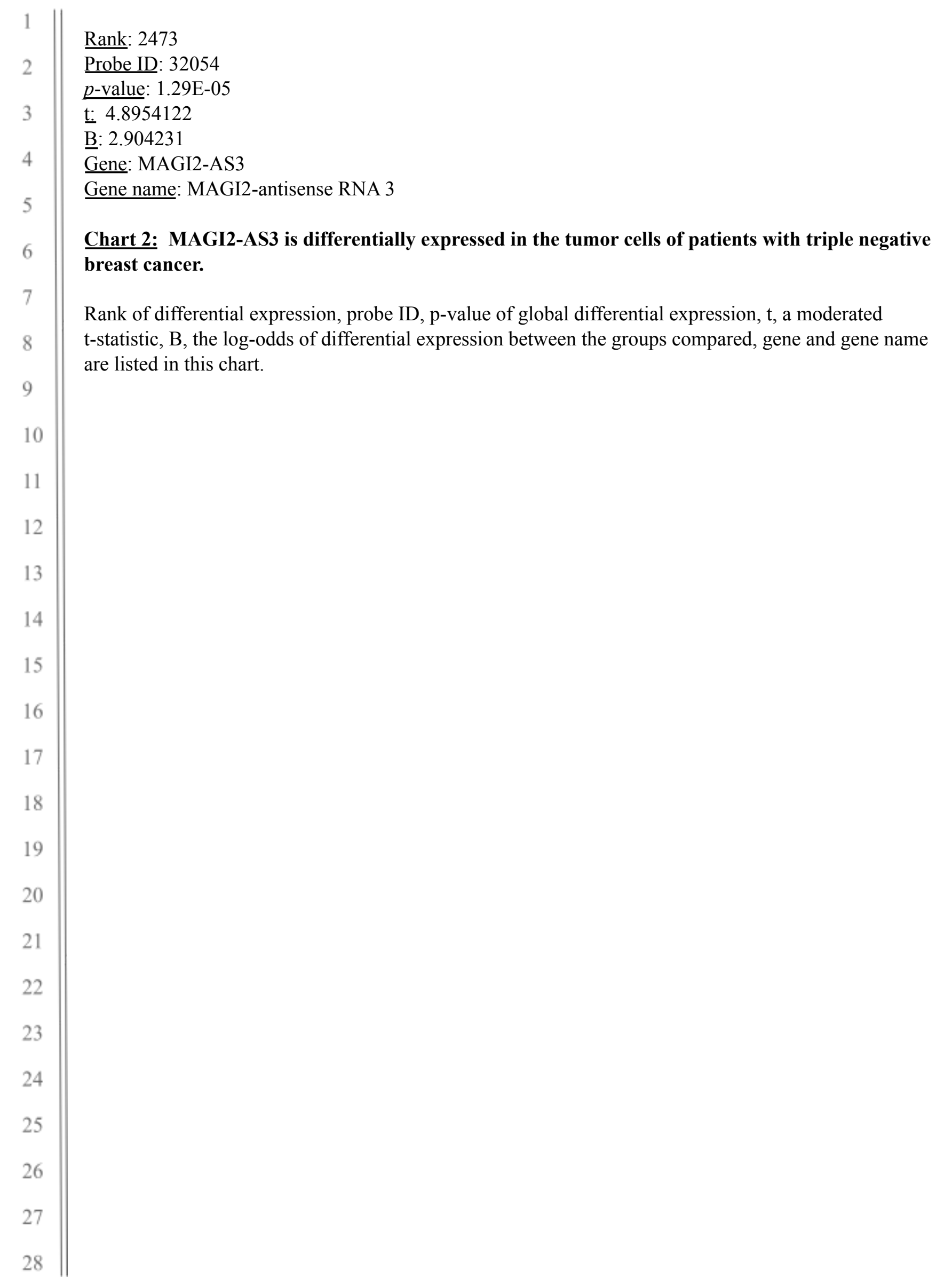




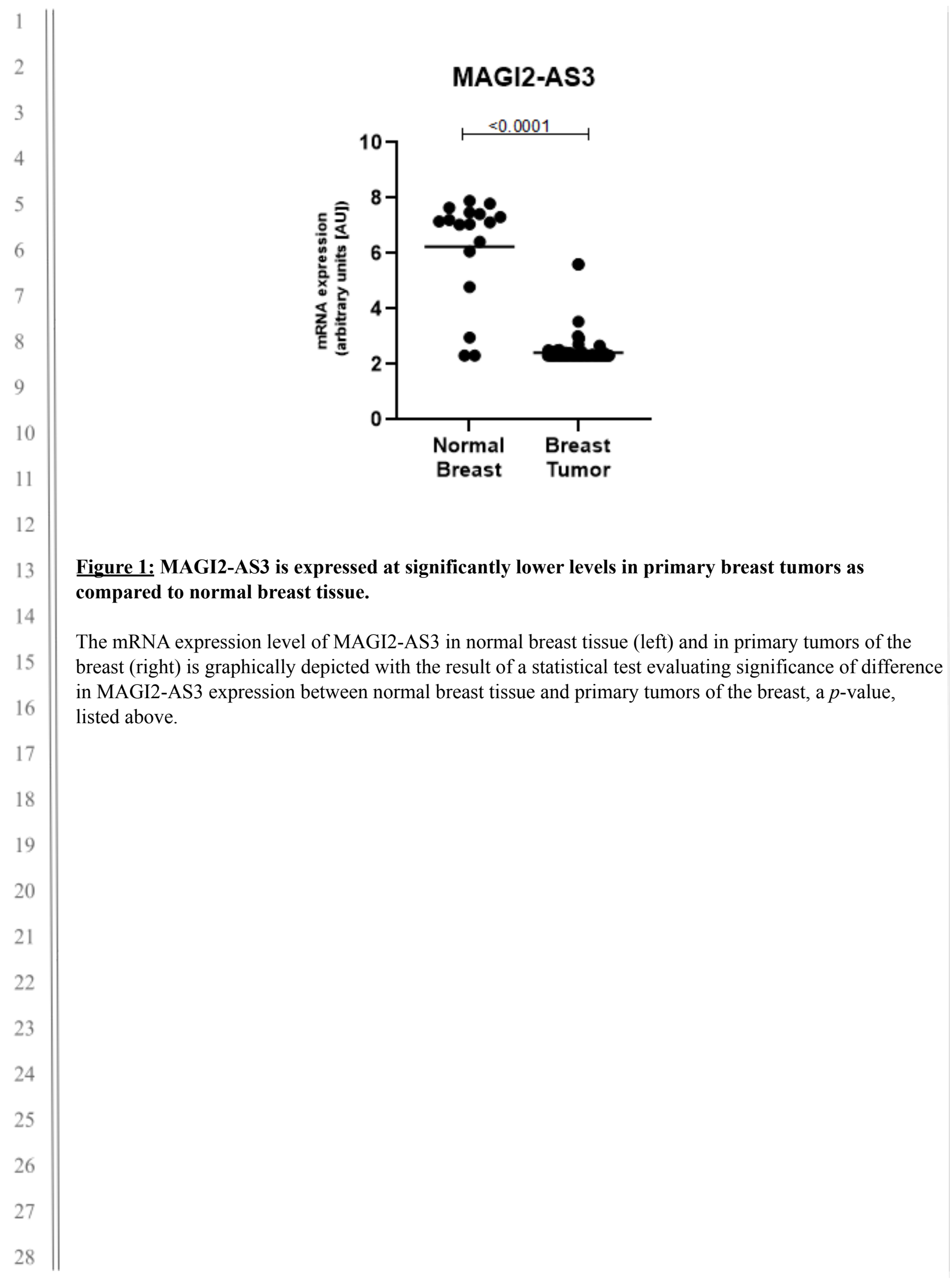




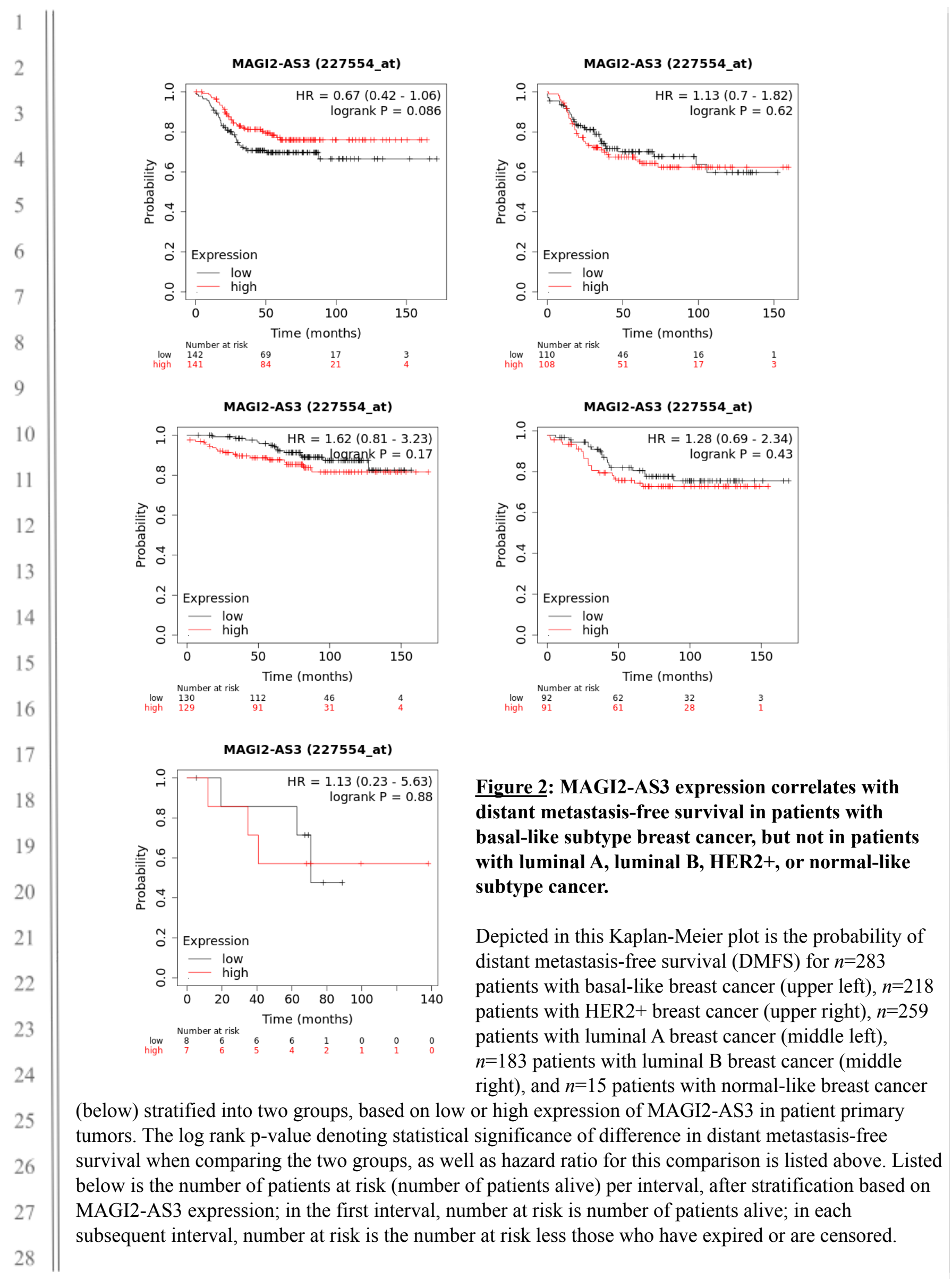

